\title{
A neutronradiography facility based on an experimental reactor
}

\author{
D. T. Thomas ${ }^{1,3}$, J. G. Fantidis ${ }^{2 *}$ and G. E. Nicolaou ${ }^{3}$
}

${ }^{1}$ European Commission, Joint Research Centre, Insitute for Energy and Transport

${ }^{2}$ Department of Electrical Engineering, Eastern Macedonia and Thrace Institute of Technology, Greece

${ }^{3}$ Laboratory of Nuclear Technology, School of Engineering, 'Democritus'University of Thrace, Xanthi, Greece

Received 29 September 2014; Accepted 4 June 2015

\begin{abstract}
A thermal Neutron Radiography (NR) facility based on the use of thermal neutron flux, generated by the PULSTAR experimental reactor, has been designed and simulated using the MCNPX code. The key objective of the proposed facility is to deliver thermal neutron flux in this range for variable values of L/D ratio, instantaneously with acceptable values for all NR parameters. Thus, with suitable aperture and collimators designs, optimization for the parameters for thermal NR was achieved, for a wide range of the collimator ratio. The short time requirements for obtaining the radiography images justify the use of the proposed system for 'real time radiography'. The system was designed under the limitation that the total Dose Equivalent Rate does not exceed at the external shield surface the limit recommended by ICRP-26.
\end{abstract}

Keywords: Neutron radiography, nuclear reactor, $M C N P X$

\section{Introduction}

In the field of non-destructive inspection, neutron radiography (NR) and X-ray radiography (also called gamma-ray radiography) are important complementary to each other techniques [1]. Although gamma-ray radiography is more commonly used, neutron radiography is anequally significant non-destructive technique and has been established as a research tool for over 70 years [2][3]. NR is commonly used in security applications, engineering studies and industry in order to determine structural defects, geology, medicine and biological research [4][5]. NR is based on two principal components. The first one is a suitable neutron beam able to provide the desired neutron flux and the second one is a device to record the image of the studying object [6]. In particular, thermal neutron radiography has been systematically developed and nowadays is commercially available. However, a problem related to the neutron imaging is the accessibility of neutron sources with high flux. The constantly increasing availability of high-intensity thermal neutron beams from nuclear reactors and neutron sources has significantly contributed to limit this restriction [7]. Nowadays, nuclear reactors, isotopic sources and accelerators comprise the necessary neutron beams.

The major goal of this study is to design a thermal NR facility based on the use of thermal neutron flux generated around the core of the PULSTAR reactor [8].The use of a nuclear reactor for generating the neutron beam has many advantages. Neutron output is constant and the neutron flux can be high which could shorten the exposure time for an object. In addition it provides excellent image quality. On the other hand, nuclear reactors have high maintenance and

* E-mail address: Fantidis@yahoo.gr ISSN: 1791-2377 (C) 2015 Kavala Institute of Technology. All rights reserved. operating costs and are not portable [9]. Both the design and the performance of the proposed facility were performed using the MCNPX Monte Carlo Code[10]. The aim is to optimize the facility design with respect to collimation and thermal neutron beam quality rendering it suitable for quality non-destructive testing.

\section{Materials and methods}

\subsection{The neutron source}

Nuclear reactors, among several types of nuclear irradiation, are able to provide high density neutron beams and consequently are widely used as neutron source in radiography applications. For the arrangement studied in this paper, the simulation was performed using as neutron source, experimental data from the output beam 5 of the reactor PULSTAR. It has a total full power output of 1$\mathrm{MW}_{\text {th. }}$. Water acts as both coolant and the moderator. An aluminum tank acts as the pool liner, which is surrounded by concrete shielding [8]. The measured total neutron flux at the beam tube 5 port entry is $\sim 2.5 \times 10^{12} \mathrm{n} \mathrm{cm}^{-2} \mathrm{sec}^{-1}$ at full power. The gamma flux is also high in the beam tube due to its direct observation of the core. The total gamma flux at the beam port entry is estimated to be $\sim 5 \times 10^{14} \gamma \mathrm{cm}^{-2} \mathrm{sec}^{-1}$ at full power [11]. Both neutrons and gammas energy spectraare derived from the previous job by Mishra and illustrated in Fig. 1 and Fig. 2 respectively.

The Y-axis in the illustrated figures shows the probability density function of the particle flux with respect to energy normalized to a maximum of unity. We can clearly observe in the neutron spectrum that the neutron beam is sufficiently thermalized. However, fast neutrons also exist and should be removed in order to approach a pure thermal beam. The gamma spectrum shows a sharp peak at $2.2 \mathrm{MeV}$, which are prompt gammas, emitted by the absorption of neutrons by hydrogen in water [11]. 


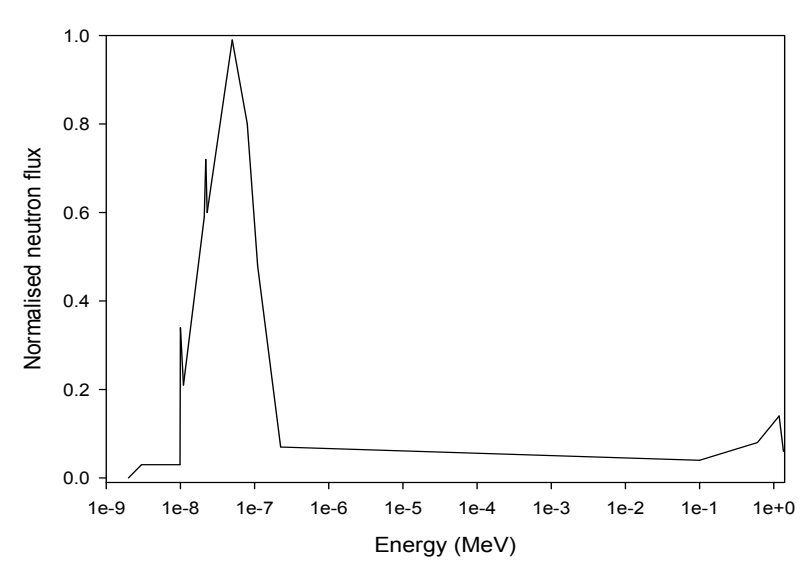

Fig 1. The neutron energy spectrum at the entry of the collimator.

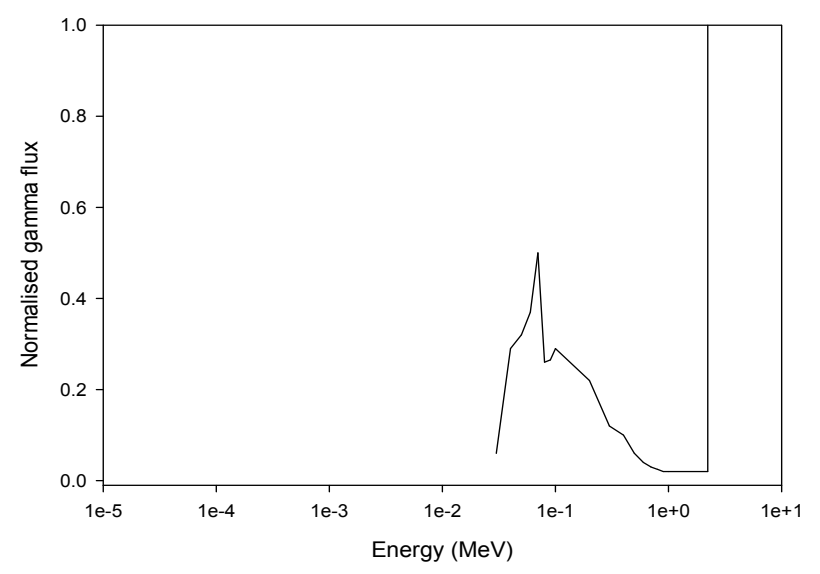

Fig 2. Gamma energy spectrum at the entry of the collimator.

\subsection{Thermal neutron collimator}

The quality of the NR imaging for a given design of the collimator is determined by the collimator ratio (L/D), which is given by the following equations:

$$
\varphi_{i}=\frac{\varphi_{\alpha}}{16\left(L_{s} / D\right)^{2}}
$$

and

$$
u_{g}=L_{f} \frac{D}{L_{s}}
$$

where $L_{f}$ is the image surface to object distance, $L_{s}$ the source to object distance, D describes the inlet aperture diaphragm, $\varphi_{\mathrm{i}}$ is the thermal neutron flux at the object plane, $\varphi_{\alpha}$ describes neutron flux at the aperture and $\mathrm{ug}_{\mathrm{g}}$ the geometric unsharpness.

Moreover, the beam divergence is a very significant measure regarding the effectiveness of the beam near its periphery. In case that the neutron beam diverges quickly, the outer section of the images generated could be significantly distorted [12]. The half-angle of the beam divergence $(\theta)$ is given by [2] $\theta=\tan ^{-1}\left(\frac{I}{2 L}\right)$

where $\mathrm{I}$ and $\mathrm{L}$ describe the maximum dimension of the image and the length of collimator respectively. The imaging quality of a system is further characterized by the Thermal Neutron Content (TNC). TNC describes the percentage of thermal neutrons within the neutron beam

$$
T N C=\frac{\text { thermal neutron flux }}{\text { total neutron flux }}
$$

Another important factor regarding the imaging quality is the $(n / \gamma)$ ration, which gives the relative intensities of the neutron $(\mathrm{n})$ and the gamma $(\gamma)$ components of the beam which typically should be [13]

$$
\frac{n}{\gamma}>10^{4} \mathrm{ncm}^{-2} \mathrm{mSv}^{-1}
$$

The flux at the image plane for research reactors is typically $10^{4}-10^{6} \mathrm{n} \mathrm{cm}^{-2} \mathrm{~s}^{-1}[14][15][16]$. The key objective of the proposed facility is to deliver thermal neutron flux in this range for variable values of $\mathrm{L} / \mathrm{D}$ ratio, instantaneously with acceptable values for all NR parameters.

\subsection{The radiography unit: design and materials}

The collimator optimization was implemented by taking into consideration the geometric unsharpness and the feasibility of reaching the maximum intensity of thermal neutrons at the image plane [17]. Fig.3 illustrates the geometric configurationof the proposed unit. The proposed radiography unit is designed in the form of two coaxial cylinders connected at one end, which is open. The left hand side cylinder incorporates the conical convergent collimator, which has a constant length, a big diameter and a small diameter of $150 \mathrm{~cm}, 15 \mathrm{~cm}$ and $6 \mathrm{~cm}$ respectively. The converged collimator aims at providing the largest possible flux of thermal neutrons in the entrance of the aperture. The walls of the cylinder are made of polyethylene with $5 \%$ boron (PE-B).

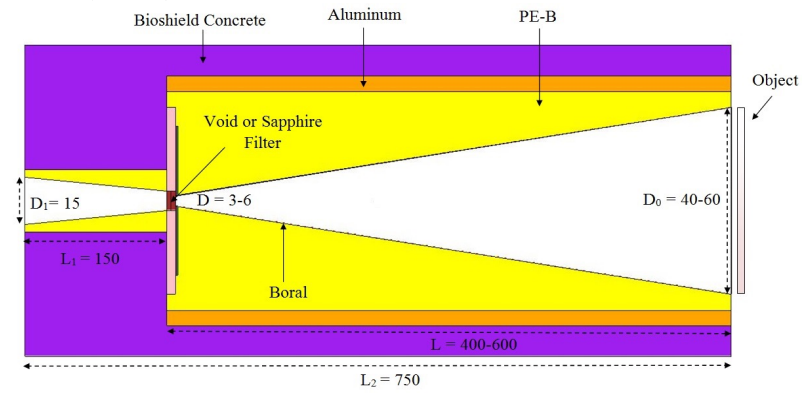

Fig 3. The collimator geometry (all dimensions in $\mathrm{cm}-$ not in scale).

The right-hand side cylinder incorporates the divergent collimator which determines, to a great extent, the quality of the image for a given source type [18]. In the optimization procedure of the collimator, the collimator length $\mathrm{L}$, the inlet aperture D and the diameter of the collimator inlet next to the image plane, $\mathrm{D}_{0}$, were altered in order to achieve the values for which the maximum thermal flux is attained in the image plane [19]. The lining of the collimator is consideredof great importance. Lining should be suitable to collimate the neutron beamin the collimator. Hence it must be designed with material that prevents stray and scattering 
neutrons from reaching the radiographed object. Materials suitable for this purpose are: boron, cadmium, gadolinium and indium [2]. In the proposed collimator, boron in the form of Boral $\left(\mathrm{B}_{4} \mathrm{C}\right)$ is used as it gets less activated facilitating this way the maintenance of the collimator [2]. Borated polyethylene (5\% boron) is used in the outside beam defining volume as it has a fairly high absorption cross-section to provide a good shielding from the neutrons, which escape from getting absorbed in the collimator lining. For the divergent collimator casing, aluminum is a very good choice. It is light weight and is relatively cheap. Also it has fairly low neutron scattering and absorption crosssection [11].

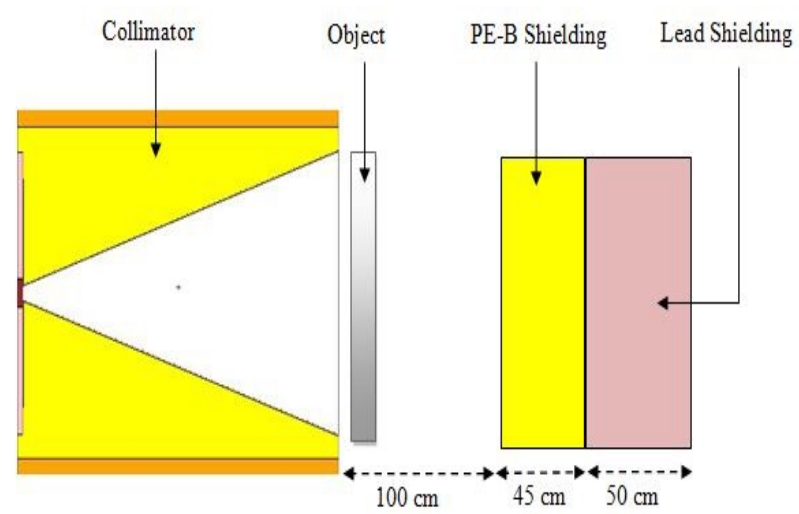

Fig 4. Simplified diagram showing the diverging collimator, the object being studied and ths shielding for both neutrons and photons ( - not in scale).

Finally the aperture is the window through which the collimated neutron beam reaches the object. The flux and the beam size at the image plane are defined, to a great extent, by the size and the shape of the aperture. Along with that, the aperture defines the geometric unsharpness in the radiograph. In addition, the $\mathrm{L} / \mathrm{D}$ ratio of the beam, where $\mathrm{L}$ defines the distance from the aperture to the image plane, depends on the aperture's length (D) [11]. The aperture consists of either three or four materials depending on whether the single sapphire $\left(\mathrm{Al}_{2} \mathrm{O}_{3}\right)$ is included in the aperture formation or not (void). The single sapphire has a variable thickness from $2.5 \mathrm{~cm}$ to $10 \mathrm{~cm}$ and is used for fast neutron filtration [20]. The aperture also contains a layer of Boral, $1 \mathrm{~cm}$ thick, which has very high thermal neutron absorption cross-section and does not get severely activated. Lead and bismuth were chosen for the gamma shielding having constant thickness $10 \mathrm{~cm}$ and $1 \mathrm{~cm}$ respectively.

The overall radiography unit layout is shielded with bioshield concrete (BC). BC is a very effective shield for both neutrons and the gamma radiation. It is a cost-effective material, which can easily be formatted into the desired shapes and dimensions. In Table 1 the chemical composition of the main materials used in the $\mathrm{BC}$ as well their corresponding density expressed in weight percent (wt. \%) are given [21].

Table 1. Chemical composition and weight percent (wt. \%) of $\mathrm{BC}$

\begin{tabular}{llllll}
\hline Element & $(\boldsymbol{\%})$ & Element & $(\boldsymbol{\%})$ & Element & $(\boldsymbol{\%})$ \\
\hline $\mathrm{H}$ & 0.6 & $\mathrm{Mg}$ & 1 & $\mathrm{~S}$ & 1 \\
$\mathrm{C}$ & 2.1 & $\mathrm{Al}$ & 3.3 & $\mathrm{Ca}$ & 13.1 \\
$\mathrm{O}$ & 50.2 & $\mathrm{Si}$ & 25.4 & $\mathrm{Fe}$ & 3.2 \\
\hline
\end{tabular}


[25]. In the case of $\mathrm{L} / \mathrm{D}=200$, the exposure time without the filter is $0.79 \mathrm{~s}$ while with the presence of the sapphire filter the time is increased at $1.13 \mathrm{~s}$. Theshort time requirements in order to obtain the radiographic images in combination with the high-flux neutron source justify the use of the proposed system for 'real time radiography' [26][27].

Table 3. The thermal NR calculated parameters for two L/D values with variable sapphire filter length.

\begin{tabular}{|c|c|c|c|c|c|c|c|c|}
\hline \multirow[b]{2}{*}{$\begin{array}{l}\text { Sapphire } \\
\text { filter } \\
(\mathrm{cm})\end{array}$} & \multicolumn{4}{|l|}{$\mathrm{L} / \mathrm{D}=75$} & \multicolumn{4}{|l|}{$\mathrm{L} / \mathrm{D}=200$} \\
\hline & $\begin{array}{l}\Phi_{\text {th }} \\
\left(\mathrm{nc} \mathrm{m}^{-2}\right. \\
\left.\mathrm{s}^{-1}\right)\end{array}$ & $\begin{array}{l}\text { TNC } \\
(\%)\end{array}$ & $\begin{array}{l}\mathrm{n} / \gamma \\
\left(\mathrm{nc} \mathrm{m}^{-2}\right. \\
\left.\mathrm{mSV}^{-1}\right)\end{array}$ & $\begin{array}{l}\text { Time } \\
\text { (s) }\end{array}$ & $\begin{array}{l}\Phi_{\text {th }} \\
\left(\mathrm{nc} \mathrm{m}^{-2}\right. \\
\left.\mathrm{s}^{-1}\right)\end{array}$ & $\begin{array}{l}\text { TNC } \\
(\%)\end{array}$ & $\begin{array}{l}\mathrm{n} / \gamma \\
\left(\mathrm{ncm}^{-2} \mathrm{~m}\right. \\
\left.\mathrm{Sv}^{-1}\right)\end{array}$ & $\begin{array}{l}\text { Time } \\
\text { (s) }\end{array}$ \\
\hline 2.5 & $3.26 \times 10^{7}$ & 95.38 & $2.41 \times 10^{6}$ & 0.30 & $1.16 \times 10^{7}$ & 95.64 & $2.33 \times 10^{6}$ & 0.86 \\
\hline 5 & $2.93 \times 10^{7}$ & 97.19 & $3.31 \times 10^{6}$ & 0.34 & $1.04 \times 10^{7}$ & 97.26 & $3.19 \times 10^{6}$ & 0.96 \\
\hline 7.5 & $2.70 \times 10^{7}$ & 98.67 & $4.57 \times 10^{6}$ & 0.37 & $0.96 \times 10^{7}$ & 98.82 & $4.43 \times 10^{6}$ & 1.04 \\
\hline 10 & $2.47 \times 10^{7}$ & 99.21 & $6.39 \times 10^{6}$ & 0.40 & $0.88 \times 10^{7}$ & 99.44 & $6.14 \times 10^{6}$ & 1.13 \\
\hline
\end{tabular}

\section{Conclusions}

A thermal NR facility based on the use of thermal neutron flux generated by the experimental reactor PULSTAR has been simulated, for radiography purposes using the MCNPX Monte Carlo code. The achieved results demonstrate that the proposed facility design can provide a wide range of the requisite parameters that are necessary for quality thermal neutron radiography imaging. The use of sapphire in the thermal neutron collimator has led to improved parameters associated with NR. The calculated thermal neutron flux $\left(\Phi_{\text {th }}\right)$ and the collimator ratio $(\mathrm{L} / \mathrm{D})$, which determines the quality of the neutron radiography imaging for a given design of the collimator, achieve values that apart from the high quality characteristics of the neutron beam demonstrate short time requirements in order to obtain the radiographic images.

\section{References}

1. Mochiki K., Murata Y., Nittoh K. (2004), Appl Radiat Isot 61:497.

2. Domanus J.C. (1987) Collimators for Thermal Neutron Radiography - An Overview, D. Reidel Publishing Company.

3. Bahnart J. (2008) Advanced Tomographic Methods in Materials Research and Engineering, Oxford University Press.

4. Fischer C.O., Stade J., Bock W., (1996) Proceedings of the fifth World Conference on Neutron Radiography, Berlin.

5. Lewis W.J., Bennett L.G.I., Chalovick T.R., Francescone O. (2000) "15th World Conference on Nondestructive Testing," Roma.

6. Fantidis, J. G., Nicolaou, G. E., \& Tsagas, N. F. (2010) J Radioanal Nucl chem 284(2):479.

7. Glasstone S. (1970), Principles of Nuclear Reactor Engineering, Macmillan and Company Ltd, 1970.

8. N. S. University, Visitors Guide to the NCSU Pulstar Reactor, Raleigh.

9. Chankow N. (2012), "Neutron Radiography," in Nondestructive Testing Methods and New Applications, INTECH, 1-29.

10. Pelowitz D.B. (2005) MCNPXTM User's Manual Version 2.5.0, Los Alamos National Laboratory, 2005.

11. Mishra, K., K. (2005) "Development of a Thermal Neutron Imaging Facility at the N.C. State University PULSTAR reactor," Raleigh.

12. Fantidis J. G. (2012) J Radioanal Nucl Chem, 293(1): 951.

13. Hawkesworth M.R. (1977), Atomic Energy Review 15 (2):169.

14. Bennett L. G. I., Chalovich, T.R., Lewis, W.J. (2005) IEEE Trans on Nucl Sci 52(1):334

15. Zawisky M., Hameed F., Dyrnjaja E., Springer J. (2010) Nucl Instrum Method Phys Res B 268 (15):2446.

16. Chankow N., Punnachaiya S., Wonglee S (2010) Appl Radiat Isot 68(4-5):662.

17. da Silva A. X., Crispim V.R. (2001) Radiat Phys and Chem 61:515.

18. Fantidis JG, Nicolaou GE, Tsagas NF (2010) Nucl Instrum Method Phys Res A 618:331.

19. da Silva A. X. Crispim VR (2001) Appl Radiat Isot 54:217

20. Mildner DFR, Lamaze GP (1998) J Appl Crystallogr 31:835.

21. Kalcheva S., Koonen E. (2005), Activation of the Concrete in the Bio Shield of ITER., SCK $\bullet$ CEN, Mol, Belgium.

22. I. C. o. R. Protection (1977) Publication 26, ICRP, Surrey.

23. Brenizer J.S., Berger H., Stebbings C.T. (1997) Rev of Sci Instrum 9(9):3371.

24. Gibbs K, Berger H, Jones T, Polansky D, Haskins J, Schneberk D, Brenizer J (1999) Flat panel imaging of thermal neutrons. In:
ASNT Fall Conference Summaries, ASNT, Columbus, pp 140142 .

25. Schillinger B., Lehnman E., Vontobel P. (2000) Physica B: Condensed Matter, 276-278:59.

26. Linfeng He, Songbai Han, Hongli Wang, Lijie Hao, Meimei Wu, Guohai Wei, Yu Wang, Yuntao Liu, Kai Sun, Dongfeng Chen (2013) Physics Procedia 43:53

27. Bücherl T., Lierse von Gostomski Ch. (2011) Nucl Instrum Method Phys Res A 651(1):175 